\title{
Pflanzliche Sekundärstoffe: Ethnobotanische Überlegungen zu ihrer Bedeutung für die menschliche Ernährung und die Medizin - Teil 3: Gemüse, Gewürze und Arzneipflanzen als Quelle wertvoller Sekundärstoffe
}

\author{
Rainer Nowack \\ Lindau, Deutschland
}

Schlüsselwörter

Sekundärstoffe · Gemüse · Gewürze · Heilpflanzen

\begin{abstract}
Zusammenfassung
Die Ernährung durch wenige domestizierte Nahrungspflanzen stellt die Zufuhr biologisch wichtiger pflanzlicher Sekundärstoffe nicht sicher. Mangelerkrankungen wie Skorbut oder Pellagra können auftreten. Mit einer gemüsereichen Kost wie rund um das Mittelmeer werden Sekundärstoffe automatisch aufgenommen. Produkte wie Knoblauch und Olivenöl emanzipieren sich aus diesem Ernährungskontext und werden separat zur Stärkung der Gesundheit eingesetzt. Eine noch weitergehende Unabhängigkeit von Speisen haben Gewürze und Arzneipflanzen. Letztere werden ohne Ernährungsbezug zur Prophylaxe und Therapie von Krankheiten eingesetzt. Gewürze und Arzneipflanzen stammen häufiger als Nahrungspflanzen aus Wildbeständen oder stehen mit diesen im genetischen Austausch. Ihre vielfach regional beschränkte Nutzung erklärt einen Trend zur Substitution wirkverwandter Pflanzen in anderen Regionen.
\end{abstract}

๑) 2016 S. Karger GmbH, Freiburg

\section{Sekundärstoffgehalt entscheidet über Verwendung als Nahrungspflanze oder Medizinalpflanze}

Der Gehalt an pflanzlichen Sekundärstoffen ist bei Nahrungspflanzen kein Domestikationsziel; ihre Selektion zielt auf einen hohen Ertrag an kalorisch verwertbaren Reservestoffen ab. Herbivore Tiere verschmähen beim Fressen Pflanzen mit hohem Sekundärstoffgehalt, wes-
Keywords

Secondary plant compounds $\cdot$ Vegetables $\cdot$ Spices $\cdot$ Herbal drugs

\section{Summary}

Secondary Plant Compounds: Ethnobotanical Reflections Regarding Their Role in Human Nutrition and in Medicine - Part 3: Vegetables, Spices and Herbal Drugs as Sources of Valuable Secondary Compounds The dietary restriction towards only a few domesticated plants does not guarantee the supply of biologically required secondary plant compounds and fosters deficiency diseases like scurvy or pellagra. A vegetable rich diet like in the Mediterranean automatically prevents such deficiencies. Products with a presumed high impact on health like garlic or olive oil are increasingly consumed on their own and independent of food. The high amounts of secondary plant compounds in spices and medicinal plants are used in well measured quantities to improve the taste of food and to prevent or treat diseases. Many spices and herbal drugs are still harvested by exploiting their wild populations. As their use is often restricted to a certain region, they are likely to be substituted by different plants of similar profile in other regions. halb diese Pflanzen auf den Weideflächen stehen bleiben [1].

Daraus folgt, dass die Deckung des Energiebedarfs durch pflanzliche Nahrung nicht automatisch die Zufuhr von Sekundärstoffen sicherstellt. Auf diese sind wir aber - zumindest in kleinen Mengen - zum Funktionieren unseres Organismus angewiesen, und wir profitieren zusätzlich, indem wir Sekundärstoffe als «borrowed molecules» z.B. zur Infektabwehr nutzen.

\section{KARGER}

Fax +497614520714

\section{๑) 2016 S. Karger GmbH, Freiburg}

Prof. Dr. med. Rainer Nowack

Dennenmoos 26, 88131 Lindau, Deutschland nowack@dialyse-lindau.de 


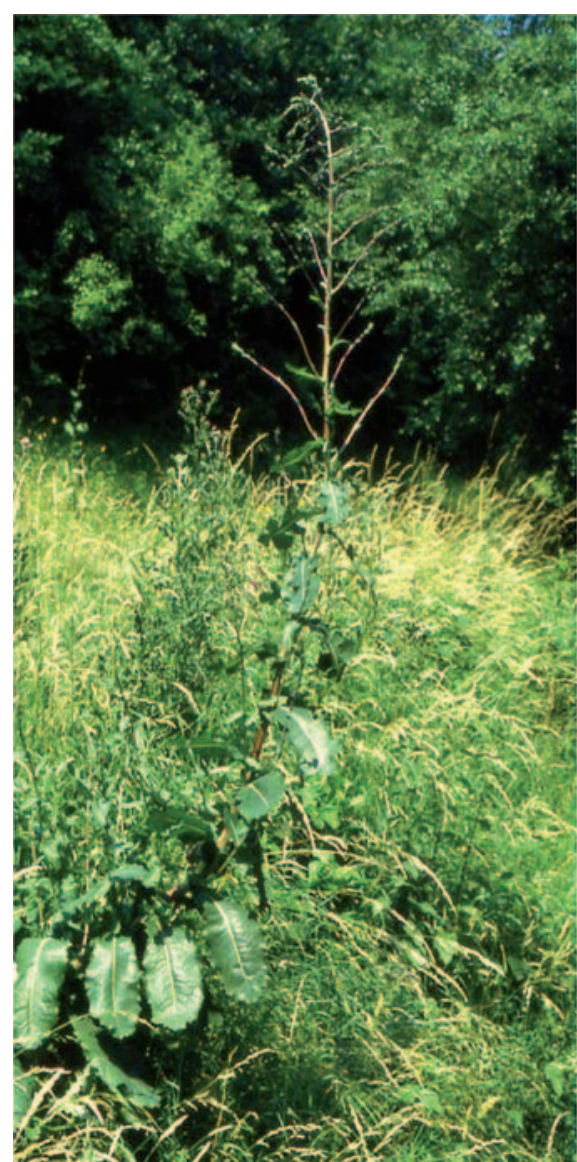
(Lactuca virosa) Leutesdorf/Rhein, 1986.
Den Gift-Lattich schätzten bereits Hippokrates und die römischen Chronisten Columella und Plinius aufgrund dessen beruhigender Wirkung. Gegen Ende des 18. Jahrhunderts verwendeten Ärzte den getrockneten Milchsaft des Gift-Lattichs als sogenanntes Lactucarium wie Opium in der Schmerztherapie. Um 1850 wurde die Pflanze an der Mosel angebaut und das Lactucarium bis nach Amerika exportiert. Besonders wirkstoffreiche Sorten wurden züchterisch gefördert $[2,3]$.

Bis zum 20. Jahrhundert waren Zubereitungen aus Gift-Lattich in Deutschland offizinell, d.h. im Deutschen Arzneibuch (DAB), gelistet. Dann gerieten sie ausser Gebrauch, bevor sie in den 1970er-Jahren von den Hippies wiederentdeckt wurden. Lactucarium wurde zum «lettuce opium». Die Ähnlichkeit mit Opium betrifft die Wirkung ebenso wie die Verarbeitung und Eindickung des weissen Milchsafts zu einem rauchfähigen Produkt. Die Forschung ist sich gar nicht sicher, ob die hypnotisierende und euphorisierende Wirkung des Lactucariums nicht überwiegend auf einem Placeboeffekt beruht. Alkaloide wie im Milchsaft des Schlafmohns lassen sich jedenfalls nicht nachweisen. Für das Lactucin, Lactucopikrin und 11ß13-Dihydrolactucin im Lactucarium sind tierexperimentell immerhin analgetische und sedierende Effekte nachvollziehbar [4].

Über das Lactucarium in Deutschland schrieb der Freiherr von Bibra im Jahre 1855 [5]:

\section{Kompass-Lattich und Gift-Lattich}

Der Kompass-Lattich (Lactuca serriola) und der GiftLattich (Lactuca virosa) (Abb. 1) bilden ein morphologisch nur schwer unterscheidbares Artenpaar. Sie enthalten einen an Triterpenen und Sekundärstoffen opioider Wirksamkeit reichen Milchsaft.

Der Kompass-Lattich ist ursprünglich eine Steppenpflanze des Orients und Nordafrikas und wurde zur Stammpflanze unseres Gartensalats (Lactuca sativa), mit dem er genetisch so weit übereinstimmt, dass man beide als eine Art auffassen kann. Kultivierte Salatpflanzen sind bereits auf 4500 Jahre alten Reliefs dargestellt. In Mitteleuropa breitete sich der krause Schnittsalat (L. sativa var. crispa) in der Zeit Karls des Grossen aus [2]; in romanischen Ländern dagegen dominierte der Bindesalat (L. sativa var. longifolia), welcher der Wildform morphologisch näher steht. Der Kopfsalat (L. sativa var. capitata) ist eine Züchtung der Neuzeit.

Wie die Wildform enthält Kultursalat im Milchsaft bittere, den typischen Salat-Geschmack ausmachende Sekundärstoffe. Die Domestikation hat den Geschmack erheblich milder werden lassen, und der Gehalt an Triterpenen ist gesunken. Dennoch gilt noch die Volksweisheit, dass eine reichhaltige abendliche Salat-Mahlzeit wie ein Schlafmittel wirken kann.
"Die Methode, das Lactucarium von Lactuca virosa zu gewinnen, welche $z u$ diesem Zwecke an mehreren Orten in grösserer Menge künstlich kultiviert wird, ist die, dass man während der Blüthezeit vor Sonnenaufgange mit einem silbernen Messer die Stengel ritzt, den ausfliessenden Milchsaft sammelt, und bei gelinder Wärme trocknet.»

Das Beispiel der Lattiche illustriert den unterschiedlichen Umgang mit Sekundärstoffen, also einerseits die Unterdrückung unerwünschter Komponenten bei Nahrungspflanzen und andererseits eine auf hohe Ausbeute zielende Förderung ihres Gehalts bei Genussmittel- und Medizinalpflanzen.

\section{Moderne Ernährungsweisen führen zu wenige Sekundärstoffe zu und machen eine Supplementation erforderlich}

Die heutige Beschränkung auf wenige Pflanzen zur Abdeckung des Kalorienbedarfs (sogenannte «staple foods») kann zu einem Mangel an gesundheitlich wertvollen Sekundärstoffen führen. Die von uns bevorzugten, auf Energiedichte hin selektionierten Pflanzen sind an Sekundärstoffen verarmt. Einige drastische, aber inzwischen durch Supplementation vermeidbare Mangelerkrankungen haben unser Bewusstsein für diese Problematik geschärft. 


\section{Klassische Mangelerkrankungen}

Auf langen Seereisen erkrankten Seeleute an Skorbut. Der Chirurg der British East India Company, John Woodall, vermutete 1617 einen Zusammenhang mit der kargen Kombüsenküche (Schiffszwieback, Pökelfleisch) und empfahl Zitronensaft auf langen Seereisen. Auch der Theologe, Techniker und Mediziner Johann Bachstrom führte Skorbut im Jahr 1734 auf einen Mangel an frischer Nahrung zurück. Captain James Cook konnte bereits Skorbut mit «Sour krout» verhindern. James Lind, ein Chirurg der British Royal Navy, wollte es genauer wissen: Er bildete unter seinen Matrosen zwei Gruppen, denen er jeweils unterschiedliche Nahrung zur Verfügung stellte. Während einer Seereise im Jahr 1747 gab Lind einigen Matrosen täglich 2 Orangen und 1 Zitrone, während die anderen Cidre, Essig, Seewasser und Schwefelsäure erhielten. In der Gruppe, die Zitrusfrüchte erhielt, blieb die Erkrankung aus. Obwohl Lind seine Beobachtung im «Treatise on the Scurvy» 1753 publizierte, setzte sich seine Erkenntnis nur schwer durch. Das mag an dem von ihm als Ersatz für frische Zitronen empfohlenen Zitronensaft-Extrakt namens «Rob» gelegen haben. Dessen Vitamin-CGehalt wurde durch das Kochen in Kupferkesseln zerstört, und er blieb folglich unwirksam. Ab 1795 schliesslich wurden bei der British Navy Zitronen oder Limetten mitgeführt. Scherzhaft nannte man die Matrosen nun «Limeys». Linds Experiment, das heute keine Ethikkommission passieren würde, gilt als Prototyp einer klinischen Studie [6].

Skorbut trat bereits vor der Ära der langen Seereisen auf, z.B. nach langen Wintern. Auch die Bedeutung pflanzlicher Nahrung zur Prophylaxe wurde schon früher erkannt. Darauf deuten Namen wie Scharbockskraut (Scharbock = Skorbut) hin, das ebenso wie die Brennnessel und weitere Kräuter im Frühjahr begierig von den Menschen konsumiert wurde. Bei der in Südwestfrankreich noch lebendigen Tradition des «la cueillette» werden hierzu sogar die Triebspitzen der giftigen Zaunrübe (Bryonia alba) geerntet [7]. In Italien wandern die wie Spargel schmeckenden Triebe des Wilden Hopfens (ital. bruscandoli) in einen Kräutersud, mit dem man Risotto und andere Speisen verfeinert. In deutschen Landen steht die berühmte Frankfurter Grüne Sosse in dieser Tradition. Abgesehen von der Pimpinelle und dem Sauerampfer stammen ihre Zutaten aus südlichen Regionen. So spekuliert man über einen römischen Ursprung dieses Rezepts. Anders ist es bei der Neun-Kräuter-Suppe, die je nach Verfügbarkeit aus einheimischen Kräutern wie Brennnessel, Gundermann, Spitzwegerich, Gänseblümchen, Löwenzahn, Vogelmiere, Scharbockskraut, Bärlauch, Giersch, Schafgarbe, Primeln, Veilchen sowie Labkraut und Taubnessel zubereitet wird. Mit der Zahl neun verbinden sich hier Zahlenmagie und Rituale aus keltisch- germanischer Zeit bis hin zum christlichen Brauchtum am Osterfest. Am Gründonnerstag legt man im Einklang mit den Fastenvorschriften für die Karwoche besonderen Wert auf grünes Gemüse.

Der Forschungsreisende Maximilian zu Wied-Neuwied erkrankte nach einem langen Winter im März 1834 in Fort Clark am Missouri an Skorbut. Er machte die Beobachtung, dass die Symptome nach dem Verzehr einer Zwiebelpflanze (Allium textile) verschwanden. Auch ein Sud aus Fichtennadeln, wie er sogar im Winter verfügbar ist, wurde als Prophylaktikum und zur Therapie von Skorbut eingesetzt.

Nach der biochemischen Entdeckung der Ascorbinsäure als Vitamin C in den 1930er-Jahren forschte man detaillierter zu dessen Bedarf. Es wurden zum Teil menschenverachtende Studien durchgeführt, unter anderem in Grossbritannien mit experimentell induziertem Skorbut an Kriegsdienstverweigern und in den 1960er-Jahren an Insassen des Iowa State Prison. Die Gefängnisinsassen entwickelten Skorbut nach 4 Wochen einer Vitamin-CDiät. Bei den britischen Kriegsdienstverweigerern dauerte es bis zu 6 Monaten. Die Skorbut-Symptome konnten durch eine Vitamin-C-Supplementation von täglich 10 mg verhindert werden [8].

In Deutschland wurde während der Zeit des Nationalsozialismus und speziell während der Kriegsjahre zur Sammlung von Hagebutten, Sanddorn, Walnüssen und Fichtennadeln aufgerufen, um daraus vitaminreiche Brotaufstriche und andere Lebensmittel herzustellen.

Andere Mangelerkrankungen treten bei einseitiger Ernährung mit einzelnen Getreidesorten auf. Basiert die Ernährung vorwiegend auf Mais- (Zea mays) oder SorghumMehl, so führt dies zur Pellagra mit charakteristischen Hauterscheinungen, Durchfällen und demenzieller Störung. Erst im 20. Jahrhundert erkannte man einen Mangel an Niacin als Ursache. Das essenzielle Niacin liegt im Mais als Niacytin vor, das vom Menschen nicht verwertbar ist, solange der Mais konventionell gemahlen wird.

Pellagra grassierte in Europa nach der Einführung des Getreides durch die Spanier im 15. Jahrhundert und in Westafrika, wo Mais erst kürzlich zum Grundnahrungsmittel wurde. Ethnien, die sich wie die Einwohner Mexikos von Mais seit Menschengedenken ernähren, vermeiden die Pellagra durch besondere Verarbeitung des Mehls. Die Körner werden in alkalischem Milieu mit gelöschtem Kalk oder Holzasche gekocht, enthülst und nass zu einem Teig vermahlen. Während dieser Prozesse wird Niacin zur Resorption verfügbar; zudem verbessern sich der Geschmack und die Backeigenschaften des Mehls. Die Technik wird als Nixtamalisierung bezeichnet und ist in $\mathrm{Oa}-$ xaca bereits um 1500 v. Chr. dokumentiert. Durch Nixtamalisierung gewonnenes Mehl wird in den Südstaaten der USA «hominy grits» und in Mexiko «masa harina» genannt [9]. 


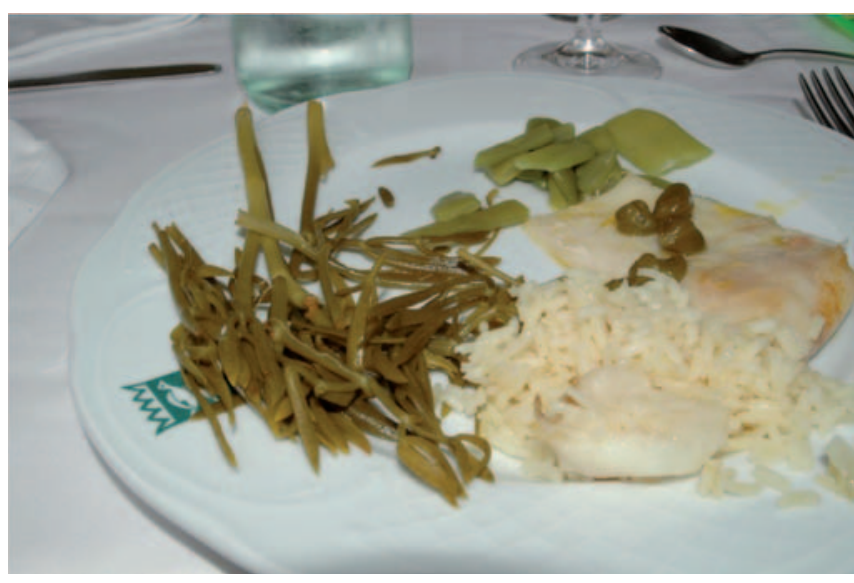

Abb. 2. Meerfenchel (Crithmum maritimum) als Wildgemüse auf Mallorca, 2006 .

Beri-Beri ist eine weitere Vitamin-Mangelerscheinung. An ihr erkranken Menschen, wenn sie sich lange Zeit beinahe ausschliesslich von geschältem Reis ernähren.

\section{Vermeidung des Mangels an pflanzlichen Sekundärstoffen durch gemüsereiche Kost}

Die Mangelerkrankungen lassen sich durch vergleichsweise geringe Zufuhren der fehlenden Komponenten verhindern, z.B. durch kleine Portionen an frischem Obst und Gemüse. Die Sekundärstoffe können ihre physiologischen Aufgaben bereits in Spuren erfüllen. Eine Ernährungsweise, die traditionell eine Vielfalt von pflanzlichen Lebensmitteln einschliesst, sichert diese Zufuhr automatisch.

Die mediterrane Ernährung ist hierfür ein viel zitiertes Beispiel. Aber lassen sich über die Vermeidung bekannter Mangelerkrankungen hinaus gesundheitliche Vorzüge dieser Ernährung nachweisen? Diese Frage ist kompliziert zu beantworten, da sich die zu untersuchenden Bevölkerungsgruppen nicht nur hinsichtlich der Ernährung, sondern in weiteren Merkmalen des Lebensstils und zusätzlich genetisch unterscheiden. Auch wenn einzelne Studien einen Vorteil hinsichtlich der Gesamtmortalität und speziell der kardiovaskulären Mortalität nachweisen konnten [10], so ist dies in der Gesamtbetrachtung aller durchgeführten Studien keinesfalls überzeugend belegt [11].

Dessen ungeachtet gilt eine gemüsereiche Kost im Allgemeinen als günstig für die Gesundheit, und diese Überzeugung prägt heute den Speiseplan vieler Haushalte.

Die Verwendung von Pflanzen rund um das Mittelmeer und generell in ländlichen Regionen wird allerdings erst durch die Einbeziehung lokal vorkommender Wildpflanzen so bereichert, dass dies von einer Stadtbevölkerung nicht nachvollzogen werden kann. Die Städter bleiben auf das beschränkte und auf Grösse und Optik hin

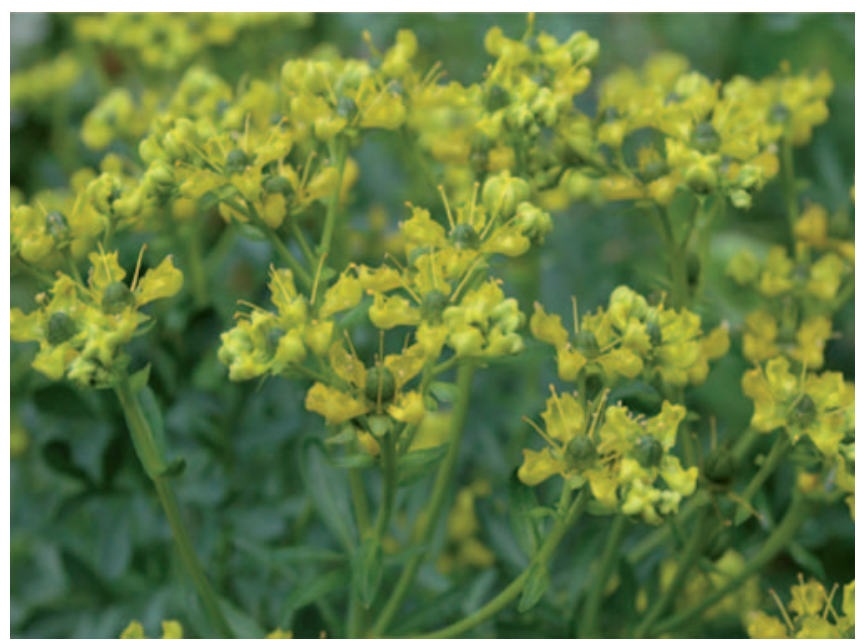

Abb. 3. Die Weinraute (Ruta graveolens) enthält intensiv riechende ätherische Öle. Sie wird als Heilmittel und in geringen Mengen als Gewürz eingesetzt.

selektionierte Gemüse-Sortiment der Supermärkte angewiesen. Generell zeichnet sich die traditionelle Verwendung von Gemüsen durch Volkstümlichkeit und Regionalität aus. Beispiele hierfür sind der in Küstenregionen vorkommende und leicht salzig schmeckende Mönchsbart (Salsola soda) oder der Meerfenchel (Crithmum maritimum) (Abb. 2). Meerfenchel bereichert als «Fonoll mari» die mallorquinische Küche und als «Finnochio marino» diejenige Südapuliens; er gilt übrigens auch als ein Skorbut-Prophylaktikum.

Gemüse werden als Beikost der «staple foods» verzehrt. Sie sind bei einem hohen Gehalt an Vitaminen, Sekundärstoffen, Mineralien und Ballaststoffen geschmackgebend und kalorienarm. Dabei teilen sie viele Eigenschaften mit Küchenkräutern und Gewürzen. Fenchel, Zwiebel, Knoblauch, Paprika und Petersilie werden sowohl als Gemüse als auch als Gewürz verwendet. Sie gelten dann als Gemüse, wenn sie eine Hauptkomponente der Mahlzeit bilden. Über die Küchenkräuter und Gewürze gibt es wiederum Übergänge zu den Arzneipflanzen. Hippokrates sowie Mediziner und Biologen der Gegenwart wollen daher zwischen Nahrung und Medizin keine scharfe Trennung vornehmen [12].

Aufgrund ernährungswissenschaftlicher und medizinischer Erforschung ihrer Sekundärstoffe werden einzelnen Produkten wie z.B. Kräutern der Provence, Olivenöl, Knoblauch, Artischocken und Rotwein innerhalb der Vielfalt mediterraner Speisen eine herausragende gesundheitsförderliche Wirksamkeit zugesprochen.

Es liegt nahe, diese Produkte aus dem ursprünglichen Ernährungskontext zu lösen, um sie in neuartigen Lebensmitteln oder Nahrungsergänzungsmitteln wie Olivenölkapseln, Knoblauch-Salz, Kräuterbutter, Spinatnudeln usw. zu konsumieren. So entstand unter anderem eine inflationäre und beliebige Verwendung von «medi- 
Abb. 4. Stempelfäden von Crocus sativus auf einem Stand des Gewürzbasars in Istanbul, 2013. Das Gewürz Safran wurde wegen seines hohen Preises häufig mit Produkten anderer Pflanzen verfälscht.

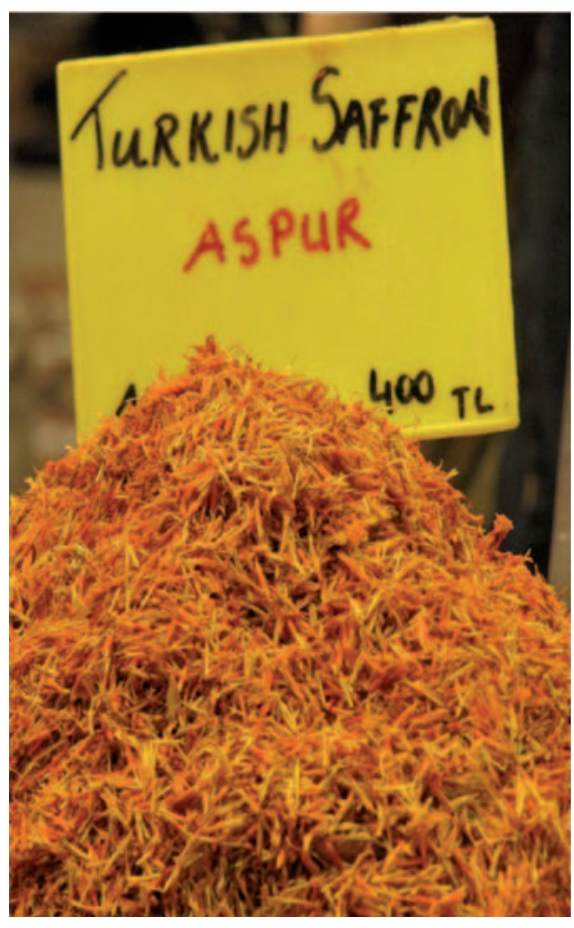

terranen» Kräutern. Mit der Mitte des 19. Jahrhunderts sich aus der Chemie emanzipierenden Ernährungswissenschaft werden immer mehr Inhaltsstoffe von Lebensmitteln erforscht und diese Erkenntnisse rasch popularisiert. So wurde man bereits als Kind der 1960er-Jahre zum Carotin-Gehalt der Karotten und einem Einfluss auf die Sehkraft belehrt, wenn man mal wieder mit dem Gemüse haderte.

\section{Gewürze}

Gewürze stehen den Medizinalpflanzen bereits recht nahe. Weinraute (Abb. 3), Muskatnuss, Safran (Abb. 4), Salbei, Ysop und Wermut sind Würz-Kräuter, die arzneilich und in vorsichtiger Dosierung zur Verfeinerung von Speisen genutzt werden. Bei übermässigem Genuss wirken ihre Inhaltsstoffe toxisch. Der Asant (Ferula assa-foetida) mit seinem extrem bitteren und beissenden, entfernt auch an Knoblauch erinnernden Geschmack ist ein Gewürz, bei dem sich zunächst alle Sinne sträuben. In Spuren setzt er in Gerichten, wie sie die indische Küche schätzt, interessante Akzente und macht sie bekömmlicher. Geringe Mengen sind in der berühmten «Worcestersauce» enthalten.

Mit Gewürzen konservierte man in der Antike und im Mittelalter Fleisch (auch Leichen). Wie erfolgreich diese Anwendungen waren, zeigen uns die heute noch gut erhaltenen Mumien [13]. Gewürze und Arzneien waren damals weitgehend identisch und wurden als «pigmenta» bezeichnet.
Abb. 5. Schmalblättriger Doppelsame (Diplotaxis tenuifolia), eine häufig ruderal vorkommende Art hier in der Normandie, 2013. Die Blätter werden als «Rukola» gegessen.

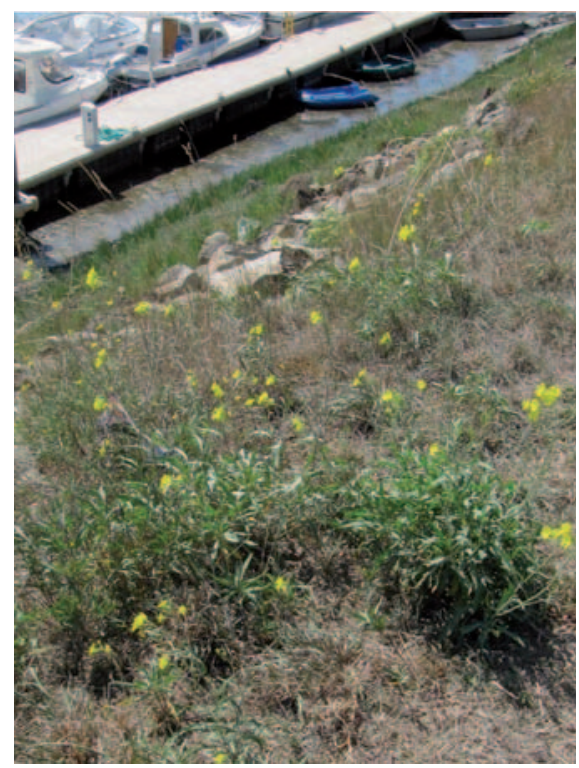

\section{Arzneipflanzen}

Während Gewürze noch einen Bezug zu Speisen haben, werden reine Arzneipflanzen von der Nahrungsaufnahme getrennt in Sondersituationen eingenommen. Zwar gibt es bei Tees einige Überschneidungen, aber für die meisten Arzneipflanzen gilt, dass für ihre Wirkung Sekundärstoffe verantwortlich sind, die unter normalen, d.h. gesunden Umständen nicht konsumiert werden würden. Ihr Geschmack ist häufig bitter oder in anderer Weise unangenehm.

Warum sich Menschen und Tiere dazu überwinden, sie im Krankheitsfall zu konsumieren, ist noch weitgehend unbekannt. Aufschlussreich sind Beobachtungen und Experimente an Insekten, an Säugetieren und speziell an Primaten [14]. Ganz offensichtlich verlassen die Tiere ihre Ernährungsgewohnheiten, wenn sie krank werden und suchen gezielt Pflanzen mit toxischen Sekundärstoffen auf. Experimente an Käfern zeigen, dass sich deren Geschmackspräferenzen bei Parasitenbefall so ändern, dass sie arzneilich wirksame Pflanzen zu fressen beginnen, die sie sonst strikt meiden [15]. Menschen wiederum, denen diese instinktive Vertrautheit mit der Pflanzenwelt verloren gegangen ist, kopieren Beobachtungen, die sie an Tieren machen.

Die Selbstmedikation von Tieren, die Zoopharmakognosie, ist ein neuer Forschungszweig, der auch für die Auswahl von Medizinalpflanzen durch die Menschen neue Erkenntnisse bringen kann. Die meisten Erkenntnisse liegen bisher zur Selbstmedikation der Menschenaffen Afrikas vor. So fressen Schimpansen giftige Vernonia-Arten zu definierten Zeitpunkten, um sich von Parasiten zu befreien. Von Afrikanischen Elefanten ist bekannt, dass sie kurz vor der Geburt Wuchsorte einer Boraginacee 


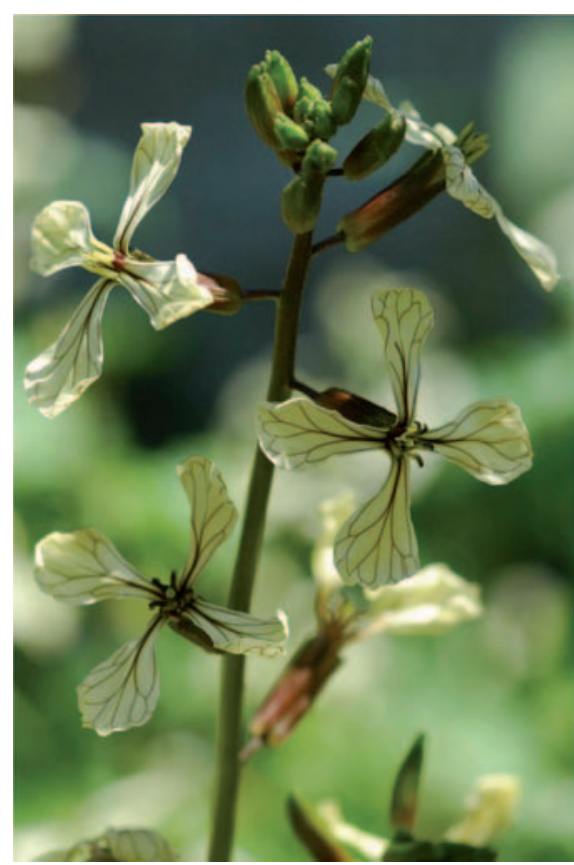
der etwas edlere «Rukola». Lindau, 2004.

aufsuchen und durch deren Verzehr die Wehen auslösen. In Kenia haben schwangere Frauen diesen «Trick» von den Elefanten übernommen.

Die Entdeckung arzneilicher Wirkungen kann auch zufällig bei akzidenteller oder anders motivierter Aufnahme des Wirkstoffs geschehen. Vermutlich geht die Verwendung von Mutterkorn (Secale cereale) als blutstillendes Prinzip, unter anderem bei Gebärmutterblutungen, auf Beobachtungen an Ergotismus-Patienten zurück. Während der Epidemien des Antoniusfeuers während des Mittelalters gab es hierzu reichlich Gelegenheit. Zuvor spielten die halluzinogenen Wirkstoffe aus dem Mutterkorn bereits bei den Initiationsfeiern der Mysterien von Eleusis eine Rolle [16]. Auch die Priester, denen die Inszenierung dieser Rituale oblag, konnten das blutstillende Wirkprinzip bereits entdeckt haben.

\section{Unterschiede der Nutzungs-/ \\ Domestikationshistorien von Gewürzen und Medizinalpflanzen gegenüber den Nahrungspflanzen}

Von den Gemüsen über die Gewürze bis zu den Arzneipflanzen treten die Sekundärstoffe und ihre Wirkungen immer deutlicher in den Vordergrund und der Ernährungsaspekt der Pflanzen fällt schliesslich weg. Mit dieser Emanzipation der funktionellen, kulinarischen und medizinischen Qualitäten der Sekundärstoffe kann der Mensch als Nutzer auch auf andere Pflanzen zurückgreifen, wenn sie gleichsinnig verwendbare Inhaltsstoffe enthalten.

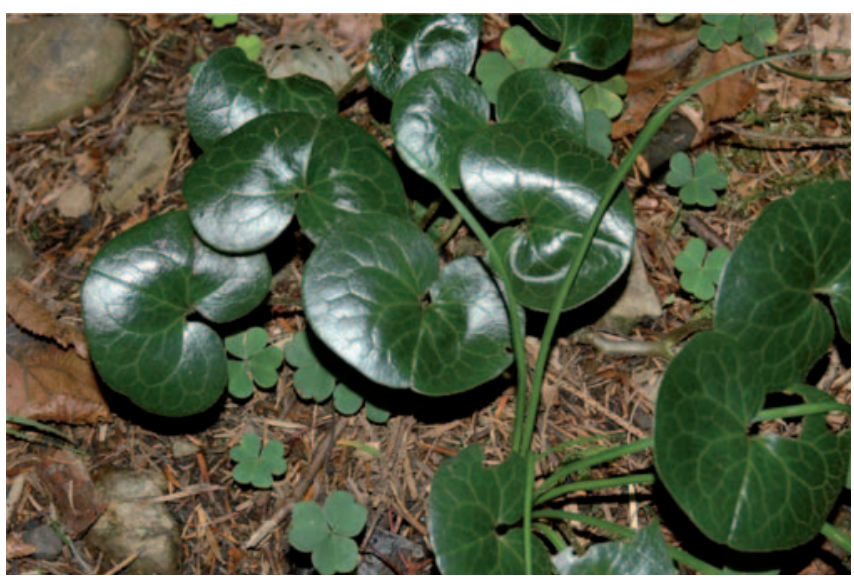

Abb. 7. Haselwurz (Asarum europaeum), Lindau, 2002. Die Haselwurz war seit der Antike eine bedeutende Heilpflanze, unter anderem zur Behandlung von Gicht. Ihre Giftigkeit durch den Gehalt an Asaron wurde erst nach und nach erkannt.

\section{Das «me too»-Prinzip: Substitution in der Pflanzennutzung}

Zur Substitution geeignete Pflanzen werden häufig entdeckt, wenn sie in direkter Nachbarschaft des Originals wachsen und versehentlich in das Erntegut gelangen. Der Ackersalat (Valerianella spec.) wuchs jahrhundertelang unbeachtet als Unkraut im Gemüsebeet, bevor man seine Nutzbarkeit erkannte. Erst seit dem 17. Jahrhundert sind sein Anbau und die Auslese von grossblättrigen Sorten dokumentiert [17].

Rukola kann von vornherein nicht auf eine botanische Art festgelegt werden, sondern ist ein Sammelbegriff für verschiedene Kreuzblütler, denen ein spezieller nussigscharfer Geschmack zu eigen ist. Rukola ist ein Trendprodukt; meist stammt er aus dem Anbau des vom Unkraut zur Kulturpflanze gewandelten Doppelsamens (Diplotaxis tenuifolia) (Abb. 5) oder der etwas schärfer schmeckenden Rauke (Eruca sativa) (Abb. 6), die bereits in klassischer Zeit als Salat und Ölpflanze genutzt wurde [18].

Unter den Gewürzen gibt es zahllose Beispiele für ähnliche Geschmacksqualitäten bei phylogenetisch nicht näher verwandten Pflanzen. Unter «Pfeffer» stehen im Gewürzregal die Arten der Gattung Piper (Piper nigrum und Piper cubeba) aus den Piperaceae, der Szechuan-Pfeffer (Zanthoxylum piperitum) aus der Familie der Rutaceae und der Peruanische Pfeffer (Schinus molle) aus den Anacardiaceae; unter «Anis» finden sich der heimische Doldenblütler Pimpinella anisum und der asiatische Sternanis (Illicium verum) aus der Familie Schisandraceae usw. Ein dem Anis ähnlicher Geschmack ist zudem im Estragon (Artemisia dracunculus) aus der Familie der Asteraceae wiederzufinden.

Allen Beispielen ist gemeinsam, dass neben einer Übereinstimmung in der Hauptgeschmacksqualität auch rele- 
Abb. 8. Kalmus (Acorus calamus) wurde als «Deutscher Ingwer» verwendet. Charlottenhofer Weiher in der Oberpfalz, 2016.

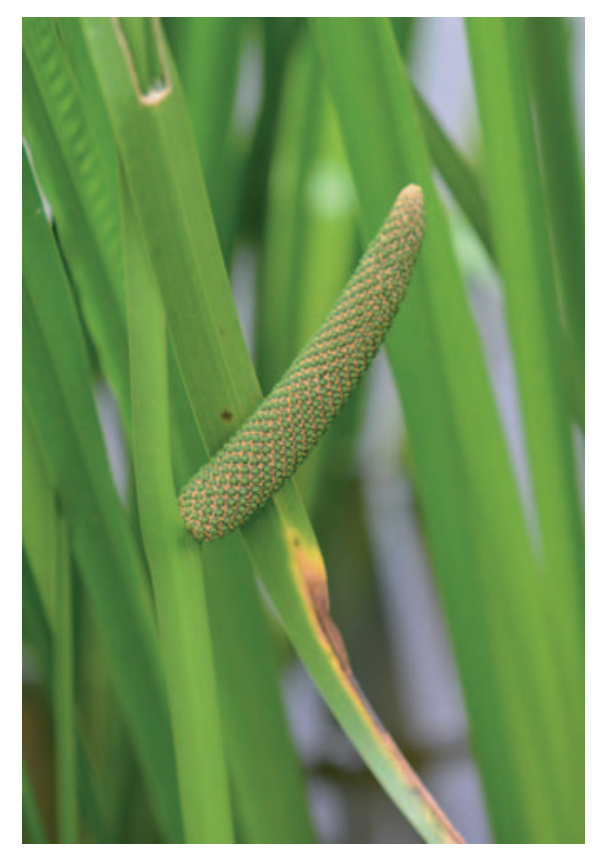

vante Unterschiede in den Sekundärstoffzusammensetzungen bestehen, die den Gewürzen ihr individuelles Profil geben. Die Würze des Echten Ingwers (Zingiber officinale) ist in der chinesischen Küche unverzichtbar, sodass bei fehlender Verfügbarkeit des Originals auf Pflanzen mit ähnlichem Geschmacksprofil zurückgegriffen wird. Zwei als Ingwer-Ersatz verwendete Pflanzen enthalten allerdings toxisches $\beta$-Asaron (Abb. 7). In Nordamerika haben die Indianer Asarum canadense als «wild ginger» genutzt. Wie alle Pflanzen der Familie Aristolochiaceae enthält er toxisches und vor allem kanzerogenes $\beta$-Asaron und Aristolochiasäure. Mit dem Kalmus (Acorus calamus) (Abb. 8) kommt ein Ingwer-Ersatz aus einer weiteren Pflanzenfamilie ins Spiel. Kandiert wird die Wurzel als «Deutscher Ingwer» gegessen, nichtsdestoweniger enthält sie Asaron. Asaron hat eine dem Meskalin verwandte Struktur und kann halluzinogen wirken. Problematischer ist allerdings die Mutagenität, und heute muss sichergestellt sein, dass nur $\beta$-Asaron-freier Kalmus in den Handel kommt. Kalmus ist eine uralte, aus Asien stammende Kulturpflanze, die sich mindestens seit dem 16. Jahrhundert in Mitteleuropa eingebürgert hat. Morphologisch sind diploide, triploide und tetraploide Formen nicht unterscheidbar. Nur die diploiden Formen sind frei von $\beta$-Asaron. Sie sind allerdings in Europa unter den eingebürgerten Vorkommen nicht verbreitet, weshalb von einer Wildernte Abstand genommen werden sollte [19].

In der Phytotherapie ist eine Substitution mutmasslich ähnlich wirkender Pflanzen eine viel geübte Praxis. In Lehrbüchern der Chinesischen Medizin wird explizit ein Kanon von Pflanzen zur Substitution für die Leitkräuter

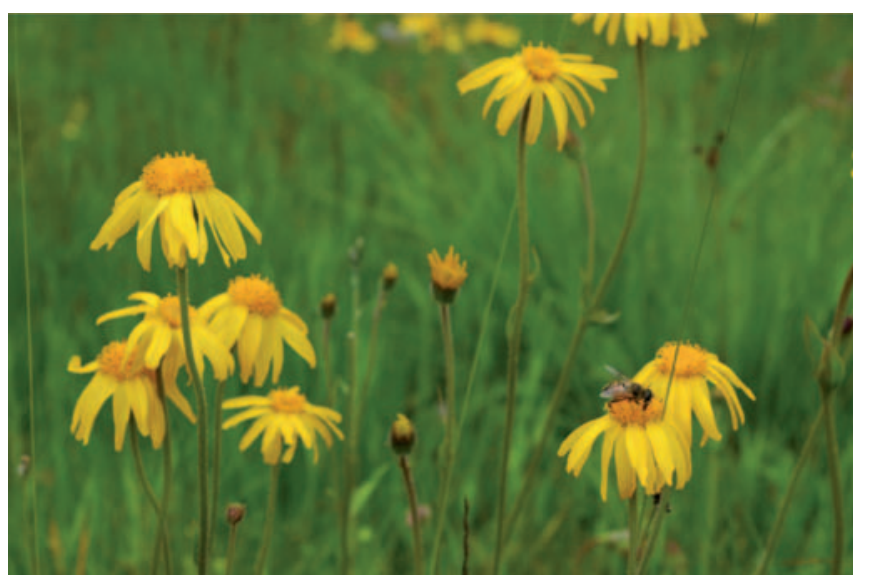

Abb. 9. Arnika (Arnica montana), Lindenberg im Allgäu, 2004.

empfohlen, wenn diese nicht zur Verfügung stehen [20]. Im riesigen, sich über mehrere Klimazonen erstreckenden chinesischen Reich müssen Phytotherapeuten auf ein jeweils sehr unterschiedliches Sortiment lokaler Heilpflanzen zurückgreifen.

Wie eine vermutlich auf Verwechslung beruhende Substitution in eine profunde Heiltradition münden kann, zeigt das Beispiel der Arnika (Arnica montana) (Abb. 9). Arnika wird in den klassischen Kräuterbüchern nicht als Heilpflanze erwähnt, dagegen aber die Ringelblume (Calendula arvensis und Calendula officinalis) (Abb. 10). Calendula war neben der Kamille die wichtigste Wundheilpflanze der Araber, Griechen und Römer. Ihre Herkunft ist unsicher; während der Antike war sie bereits im Mittelmeerraum verbreitet, und mittlerweile kommt sie verwildert überall in Europa vor. Die (für Botaniker allerdings nur entfernte) Ähnlichkeit ihrer Blüten hat die Heilkundigen wohl dazu bewogen, Arnika ähnlich wie die Ringelblume zur Wundheilung einzusetzen, und das überraschenderweise mit guten Resultaten. Seit dem Mittelalter werden beide Pflanzen als gleichwertige Wundheilmittel empfohlen [21].

Substitutionen von pflanzlichen Heilmitteln können aber auch verhängnisvolle Folgen haben, wenn die Anwender nicht ausreichend mit ihnen vertraut sind. Die chinesische Pharmakopöe lässt die Substitution von Spezies sehr verschiedener Toxizität zu, beispielsweise des mild diuretisch wirksamen Han Fang ji = Stephania tetranda (Menispermaceae) durch das toxische Guang Fang ji = Aristolochia fangchi. Als diese chinesischen Heilpflanzen in einer belgischen Klinik als Fasten-Regimen eingesetzt wurden, erkrankten junge Frauen schwer an Nierenversagen und an Karzinomen. Sie hatten eine unzulässig hohe Dosis von Aristolochia erhalten, dessen mutagene und nephrotoxische Wirkung bereits bekannt war [22]. 


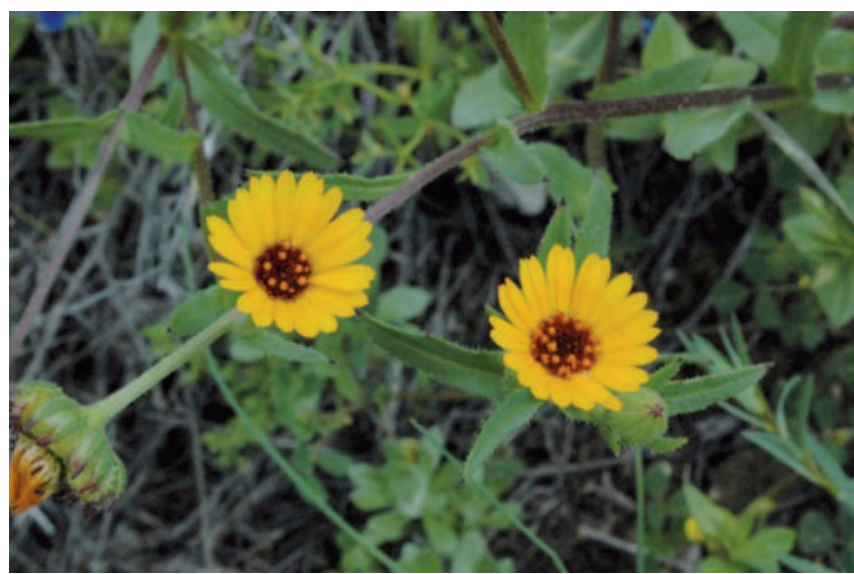

Abb. 10. Ringelblume (Calendula arvensis) auf Zypern, 2009.

\section{Arzneipflanzen, Genussmittel und Gewürze stammen häufig aus Wildsammlungen oder extensiven Kulturen}

Die Substitution eines raren Originals durch besser verfügbare Pflanzen mit vergleichbaren Qualitäten kann eine Strategie zur Schonung bedrohter Arten sein. Der Ginseng (Panax ginseng) war als sogenanntes Adaptogen während des 20. Jahrhunderts weltweit so gefragt, dass die Ernte aus Wildsammlungen nicht mehr ausreichte. Die Gefährdung des Koreanischen Ginsengs hat die Etablierung seines aufwendigen Anbaus gefördert, ebenso wie die Erforschung wirkungsverwandter Pflanzen anderer Regionen. Dazu zählen der Amerikanische Ginseng $(\mathrm{Pa}-$ nax quinquefolius) und der Sibirische Ginseng (Eleutherococcus senticosus), zu dem während Stalins Imperium intensiv geforscht wurde. Der Wurzeldroge aus Sibirischem Ginseng sagt man eine sagenhafte leistungssteigernde Wirkung nach. Nicht nur sowjetische Olympiateams wurden damit fit gemacht, auch die Opfer der Reaktorkatastrophe von Tschernobyl erhielten sie zur Vorbeugung von Strahlenschäden. So konnten sich die Bestände des Koreanischen Ginsengs stabilisieren [23].

Ein berühmtes Beispiel einer Ausrottung durch Übersammlung ist das legendäre Silphium, ein wohl in der Antike vorwiegend medizinisch genutzter Doldenblütler aus der Gattung Ferula (Abb. 11). Die Bestände fielen einer unkontrollierten Wildernte zum Opfer, bevor man noch daran dachte, den Arterhalt durch Kulturen zu sichern. Heute würde man von «Erhaltungskultur» sprechen, die für bedrohte Arten und gefährdete Kultursorten gängige Praxis ist. Antike Autoren wie Plinius der Ältere und Theophrast geben übereinstimmend an, dass Silphium nur in einem kleinen Areal der Kyrenaika, dem heutigen Libyen, wuchs. Sie berichten auch davon, wie die Nachfrage sprunghaft anwuchs, bevor die Bestände erschöpft waren.
Abb. 11. Ferula communis in Famagusta, Zypern, 2009. Zur gleichen Gattung hat vermutlich das legendäre «Silphium» gehört, das bereits in der Antike ausgerottet wurde.

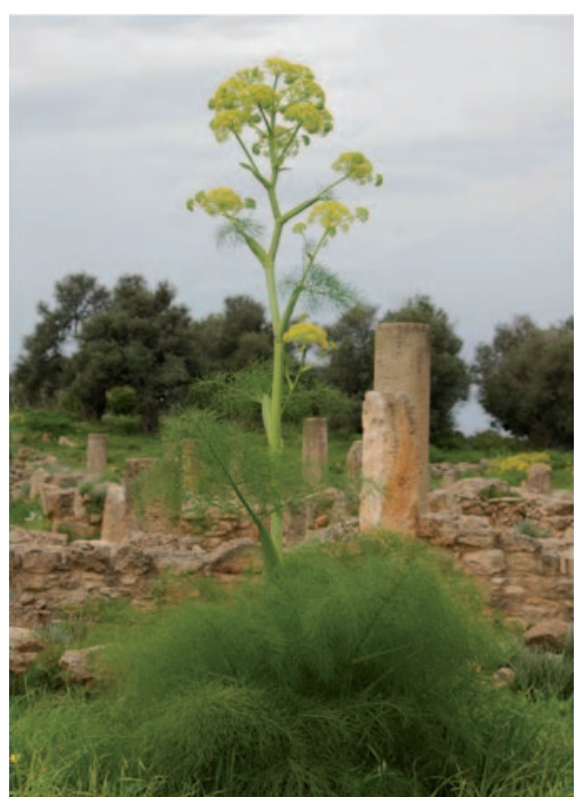

Generell kann die gegenüber Nahrungspflanzen geringere Nachfrage bei Gewürzen und Heilkräutern auch heute noch oft durch Wildsammlungen gedeckt werden (mündliche Auskunft von Dr. Rumyana V. Todorova von Dr. Todorova Consulting am 10.10.2014). Die Vorzüge eines kontrollierten Anbaus hinsichtlich einer gleichbleibenden Wirkstoffzusammensetzung und -qualität sind allerdings so evident, dass dieser sicher zukünftig wachsen wird [24].

\section{Schluss}

Die Versorgung mit Sekundärstoffen ist heute weitgehend von der Kalorienzufuhr abgekoppelt und erfolgt über kulinarische Beilagen, Gewürze, Nahrungsergänzungsmittel und Arzneien. Mit der Produktion und Anwendung von Pflanzen, die wir wegen ihres Gehalts an Sekundärstoffen konsumieren, sind andere Berufe als im Nahrungsmittelsektor befasst. Der Winzer ist zwar ähnlich wie der Landwirt organisiert, muss sich aber im Interesse seiner Kundschaft dezidiert mit der Pflege der Eigenheiten seiner Reben ohne vordergründig ertragssteigernde Ambitionen befassen. Beim Wein und bei weiteren pflanzlichen Genussmitteln huldigt der Verbraucher den durch die Sekundärstoffe mitbestimmten Nuancen in einer Weise, die nicht recht zum Konsumverhalten bei der alltäglichen Ernährung passt.

Noch deutlicher ist die Eigenständigkeit der Berufe, die mit der medizinischen Anwendung von Pflanzen befasst sind. Arzneipflanzen werden noch immer gesammelt und nur in kleinem Massstab kultiviert. Während viele Jahrhunderte lang bis zu 90\% der Menschen in der landwirt- 
schaftlichen Nahrungsmittelproduktion arbeiteten, waren mit der Heilkunde immer nur wenige Spezialisten befasst. Vielleicht ändert sich dies, denn nach einer Bedeutungskrise der Phytotherapie Mitteleuropas durch die aufkommende wissenschaftliche Medizin und Pharmazie im 19. Jahrhundert gibt es längst ein wiedererwachtes Interesse an der Kräutermedizin.

\section{Disclosure Statement}

Keine Interessenkonflikte.

\section{Literatur}

1 Villalba JJ, Provenza FD: Self-medication and homeostatic behaviour in herbivores: learning about the benefits of nature's pharmacy. Animal 2007;1:1360-1370.

2 Körber-Grohne U: Nutzpflanzen in Deutschland: Kulturgeschichte und Biologie. Stuttgart, Theiss, 1987

3 Wagenitz G (Hrsg): Illustrierte Flora von Mitteleuropa. Pteridophyta, Spermatophyta. Begründet von Gustav Hegi. 2. überarbeitete und erweiterte Auflage. Band VI. Teil 4: Angiospermae, Dicotyledones 4 (Compositae 2, Matricaria - Hieracium). Berlin/Hamburg, Paul Parey, 1987, pp 1122-1124 (revidierter Nachdruck der 1. Auflage (Band VI/2 von 1929) mit Nachtrag).

4 Wesołowska A, Nikiforuk A, Michalska A, Kisiel W, Chojnacka-Wójcik E: Analgesic and sedative activities of lactucin and some lactucin-like guaianolides in mice. J Ethnopharmacol 2006; 107:254-258.

5 Bibra E: Die narkotischen Genussmittel und der Mensch. Nürnberg, Wilhelm Schmid, 1855 .

6 James Lind: A Treatise on the Scurvy. London, 1753.

7 Mabey R: Weeds: In Defense of Nature's Most Unloved Plants. New York, Harper Collins, 2010 .

8 Pemberton J: Medical experiments carried out in Sheffield on conscientious objectors to military service during the 1939-45 war. Int J Epidemiol 2006;35:556-558.
9 Ram S, Mishra B: Cereals: Processing and Nutritional Quality. New Delhi, New India Publishing Agency, 2010.

10 Knoops KT, de Groot LC, Kromhout D, et al: Mediterranean diet, lifestyle factors, and 10 year mortality in elderly European men and women: the HALE project. JAMA 2004;292: 1433-1439.

11 Rees K, Hartley L, Flowers N, et al: 'Mediterranean' dietary pattern for the primary prevention of cardiovascular disease. Cochrane Database Syst Rev 2013;8:CD009825.

12 Ren Z-X, Wang H, Bernhardt P, Li D-Z: Insect pollination and self-incompatibility in edible and/or medicinal crops in southwestern China, a global hotspot of biodiversity Am J Bot 2014;101:1700-1710.

13 Niederer M: Der St. Galler Botanicus: ein frühmittelalterliches Herbar. Bern, Peter Lang, 2005.

14 Huffman MA: Animal self-medication and ethno-medicine: exploration and exploitation of the medicinal properties of plants. Proc Nutr Soc 2003;62:371-381.

15 Singer MS, Mace KC, Bernays EA: Self-medication as adaptive plasticity: increased ingestion of plant toxins by parasitized caterpillars PLoS One 2009; 4:e4796.

16 Wasson RG, Hofmann A, Ruck CAP: Der Weg nach Eleusis. Das Geheimnis der Mysterien. Frankfurt/M., Insel, 1984.

17 Schwanitz F: Die Evolution der Kulturpflanzen. München, Bayerischer Landwirtschaftverlag, 1967.
18 Morales M, Janick J: Arugula: a promising specialty leaf vegetable; in Janick J, Whipkey W (eds): Trends in New Crops and New Uses. Alexandria, VA, ASHS, 2002.

19 Koul O, Smirle MJ, Isman MB: Asarones from Acorus calamus L. oil. J Chem Ecol 1990;16:1911-1920.

20 Bensky D, Gamble A: Chinese Herbal Medicine. Materia Medica. Revised edition. Seattle, WA, Eastland, 1993.

21 Klaas, CA, Wagner G, Laufer S, Sosa S, Loggia $\mathrm{RD}$, Bomme $\mathrm{U}$, Merfort I: Studies on the anti-inflammatory activity of phytopharmaceuticals prepared from Arnica flowers Planta Med 2002;68:385-391.

22 Vanherweghem JL, Depierreux M, Tielemans $\mathrm{C}$, et al: Rapidly progressive interstitial renal fibrosis in young women: association with slimming regimen including Chinese herbs. Lancet 1993;341:387-391.

23 Zhuravlev Y, Koren O, Reunova G, Muzarok T, Gorpenchenko T, Kats I, Khrolenko Y: Panax ginseng natural populations: their past, current state and perspectives. Acta Pharmacol Sin 2008;29:1127-1136.

24 Canter PH, Thomas H, Ernst E: Bringing medicinal plants into cultivation: opportunities and challenges for biotechnology. Trends $\mathrm{Bi}$ otechnol 2005;23:180-185. 